\title{
L-Fuzzy Congruences and L-Fuzzy Kernel Ideals in Ockham Algebras
}

\author{
Teferi Getachew Alemayehu, ${ }^{1}$ Derso Abeje Engidaw $\mathbb{D}^{2},{ }^{2}$ and Gezahagne Mulat Addis $\mathbb{D}^{2}$ \\ ${ }^{1}$ Department of Mathematics, Debre Berhan University, Debre Berhan, Ethiopia \\ ${ }^{2}$ Department of Mathematics, University of Gondar, Gondar, Ethiopia \\ Correspondence should be addressed to Gezahagne Mulat Addis; buttu412@yahoo.com
}

Received 25 February 2021; Revised 29 May 2021; Accepted 1 June 2021; Published 19 June 2021

Academic Editor: Basil Papadopoulos

Copyright (C) 2021 Teferi Getachew Alemayehu et al. This is an open access article distributed under the Creative Commons Attribution License, which permits unrestricted use, distribution, and reproduction in any medium, provided the original work is properly cited.

In this paper, we study fuzzy congruence relations and kernel fuzzy ideals of an Ockham algebra $(A, f)$, whose truth values are in a complete lattice satisfying the infinite meet distributive law. Some equivalent conditions are derived for a fuzzy ideal of an Ockham algebra $A$ to become a fuzzy kernel ideal. We also obtain the smallest (respectively, the largest) fuzzy congruence on $A$ having a given fuzzy ideal as its kernel.

\section{Introduction}

Zadeh [1] introduced the concept of fuzzy sets, which has been found to be very useful in diversely applied areas of science and technology. In the last two decades, several articles have been written on the application of fuzzy sets. For instance, in medical diagnosis, Kaur and Chaira [2] proposed a novel fuzzy clustering approach that enhances the quality of vague CT scan/MRI image before segmentation. In addition, many authors (e.g., [3-6]) applied the theory of fuzzy sets in decision-making. Furthermore, the theory of fuzzy sets has been conveniently and successfully applied in abstract algebra. The study of fuzzy subalgebras of various algebraic structures has been started after Rosenfeld wrote his seminal paper [7] on fuzzy subgroups. His paper has provided sufficient motivation to researchers to study fuzzy subalgebras of different algebraic structures. For instance, fuzzy ideals and fuzzy filters of MS-algebras [8-10], some generalizations of fuzzy ideals in distributive lattices [11-13], fuzzy ideals and fuzzy filters of partially ordered sets $[14,15]$, and fuzzy ideals of universal algebras [16-18] are some of recent works on fuzzy subalgebraic structures.

As an extension of Zadeh's fuzzy set theory [1], Atanassov [19] introduced the intuitionistic fuzzy sets (IFS), characterized by a membership function and a nonmembership function. Further investigation has been made by other scholars to apply the theory of intuitionistic fuzzy sets in the class of BG-algebras [20], B-algebras [21], and BCK-algebras [22] as well.

A fuzzy congruence relation on general algebraic structures is a fuzzy equivalence relations which is compatible (in a fuzzy sense) with all fundamental operations of the algebra. The notions of fuzzy congruence relations were studied in various algebraic structures: in semigroups (see $[23,24]$ ), in groups, rings, and semirings (see [25-30]), in modules and vector spaces (see $[31,32]$ ), in lattices (see $[33,34]$ ), in almost distributive lattices and MS-algebras (see $[35,36]$ ), and, more generally, in universal algebras (see [37-39]).

The notion of Ockham algebras was initially introduced by Berman [40] in 1977. In simple terminology, an Ockham algebra is a bounded distributive lattice equipped with a dual endomorphism. Blyth and Silva [41] have studied and characterized kernel ideals in Ockham algebra. The purpose of this paper is to apply the theory of $L$-fuzzy sets in the class of Ockham algebras, where $L$ is a complete lattice satisfying the infinite meet distributive law:

$$
a \wedge \sup S=\sup \{a \wedge x: x \in S\},
$$


for any $a \in L$ and $S \subseteq L$. To be specific, we study $L$-fuzzy congruences and $L$-fuzzy kernel ideals of Ockham algebras and investigate their properties. We also derive some equivalent conditions for every $L$-fuzzy ideal of an Ockham algebra $A$ to become an $L$-fuzzy kernel ideal. We give an internal characterization for the smallest and the largest $L$-fuzzy congruences on $A$ having a given $L$-fuzzy ideal as a kernel.

\section{Preliminaries}

This section contains some basic definitions and results which will be used the sequel.

Definition 1 (see [42]). An Ockham algebra is an algebra $(A ; \wedge, \vee, f, 0,1)$ of type $(2,2,1,0,0)$ in which $(A ; \wedge, \vee, 0,1)$ is a bounded distributive lattice and $x \mapsto f(x)$ is a unary operation on $A$ such that $f(0)=1, f(1)=0$, and the following de Morgan laws hold

$$
\begin{aligned}
(\forall x, y \in A) f(x \vee y) & =f(x) \wedge f(y), \\
f(x \wedge y) & =f(x) \vee f(y) .
\end{aligned}
$$

For simplicity, we denote an Ockham algebra $(A ; \wedge, \vee, f, 0,1)$ by a pair $(A, f)$.

Definition 2 (see [42]). A congruence relation on an Ockham algebra $(A, f)$ is a lattice congruence $\vartheta$ on $A$ such that $x, y \in A,(x, y) \in \mathcal{\vartheta} \Rightarrow(f(x), f(y)) \in \vartheta$.

Definition 3 (see [41]). By an ideal of an Ockham algebra $(A, f)$, we mean an ideal of $A$ as a distributive lattice. Moreover, an ideal $I$ of an Ockham algebra $A$ is called a kernel ideal if there exists a congruence $\vartheta$ on $(A, f)$ such that

$$
I=\vartheta(0)=\{x \in A:(x, 0) \in \mathcal{\vartheta}\} .
$$

By an $L$-fuzzy subset $\mu$ of a nonempty $X$, we mean a mapping from $X$ into $L$. The set of all $L$-fuzzy subsets of $X$ is denoted by $L^{X}$.

Definition 4 (see [43]). Let $\mu, \sigma$ in $L^{X}$. Then, the Cartesian product of $\mu$ and $\sigma$, denoted by $\mu \times \sigma$, is defined by, for all $x, y \in X$,

$$
(\mu \times \sigma)(x, y)=\mu(x) \wedge \mu(y) .
$$

The union and intersection of any family $\left\{\mu_{i}\right\}_{i \in \Delta}$ of $L$-fuzzy subsets of $X$, respectively, denoted by $\cup_{i \in \Delta} \mu_{i}$ and $\cap_{i \in \Delta} \mu_{i}$, are defined by

$$
\begin{aligned}
& \left(\bigcup_{i \in \Delta} \mu_{i}\right)(x)=\sup _{i \in \Delta} \mu_{i}(x), \\
& \left(\bigcap_{i \in \Delta} \mu_{i}\right)(x)=\inf _{i \in \Delta} \mu_{i}(x),
\end{aligned}
$$

for all $x \in X$, respectively.
Definition 5 (see [44]). For any $\mu$ and $\sigma$ in $L^{X}$, define a binary relation " $\subseteq$ " on $L^{X}$ by

$$
\mu \subseteq \sigma \text { if and only if } \mu(x) \leq \sigma(x) \text {, for all } x \in X .
$$

It can be easily verified that $\subseteq$ is a partial order on the set $L^{X}$ of $L$-fuzzy subsets of $X$ and the poset $\left(L^{X}, \subseteq\right)$ forms a complete lattice, in which, for any $\left\{\mu_{i}\right\}_{i \in \Delta} \subseteq L^{X}$,

$$
\begin{aligned}
& \sup _{i \in \Delta} \mu_{i}=\bigcup_{i \in \Delta} \mu_{i}, \\
& \inf _{i \in \Delta} \mu_{i}=\bigcap_{i \in \Delta} \mu_{i} .
\end{aligned}
$$

The partial ordering " $\subseteq$ " is called the pointwise ordering.

For $\mu \in L^{X}$ and $\alpha \in L$, the set,

$$
\mu_{\alpha}=\{x \in X: \mu(x) \geq \alpha\},
$$

is called the $\alpha$-level subset of $\mu$, and for each $x \in X$, we have

$$
\mu(x)=\bigvee\left\{\alpha \in L: x \in \mu_{\alpha}\right\} .
$$

For any $\alpha \in L$, we write $\bar{\alpha}$ to denote the constant $L$-fuzzy subset of $X$ which maps every element of $X$ onto $\alpha$.

Definition 6 (see [7]). Let $f$ be a function from $X$ into $Y$, and let $\mu$ be an $L$-fuzzy subset of $X$. Then, the image of $\mu$ under $f$, denoted by $f(\mu)$, is an $L$-fuzzy subset of $Y$ given by, for all $y \in Y$,

$$
f(\mu)(y)= \begin{cases}\sup \left\{\mu(x): x \in f^{-1}(y)\right\}, & \text { if } f^{-1}(y) \neq \varnothing, \\ 0, & \text { otherwise. }\end{cases}
$$

The preimage of $\sigma$ under $f$, symbolized by $f^{-1}(\sigma)$, is an $L$-fuzzy subset of $X$ and

$$
f^{-1}(\sigma)(x)=\sigma(f(x)) \quad \text { for all } x \in X .
$$

Definition 7 (see [33]). An $L$-fuzzy subset $\mu$ of a lattice $X$ with 0 is said to be an $L$-fuzzy ideal of $X$ if $\mu(0)=1$ and $\mu(a \vee b)=\mu(a) \wedge \mu(b)$, for all $a, b \in X$.

Dually, an $L$-fuzzy subset $\mu$ of a lattice $X$ with 1 is said to be an $L$-fuzzy filter of $X$ if $\mu(1)=1$ and $\mu(a \wedge b)=\mu(a) \wedge \mu(b)$, for all $a, b \in X$.

An $L$-fuzzy ideal (respectively, filter) $\mu$ of $X$ is said to be proper if it is not a constant map $\overline{1}$. By an $L$-fuzzy binary relation on a nonempty set $X$, we mean an $L$-fuzzy subset of $X \times X$. For an $L$-fuzzy binary relation $\Theta$ on $X$ and each $\alpha \in L$, the set,

$$
\Theta_{\alpha}=\{(x, y) \in X \times X: \Theta(x, y) \geq \alpha\},
$$

is called the $\alpha$-level binary relation of $\Theta$ on $X$.

Definition 8 (see [45]). An $L$-fuzzy relation $\Theta$ on a nonempty set $X$ is said to be

(1) Reflexive if $\Theta(x, x)=1$, for all $x \in X$

(2) Symmetric if $\Theta(x, y)=\Theta(y, x)$, for all $x, y \in X$ 
(3) Transitive if, for each $x, y \in X, \Theta(x, y) \geq \Theta(x, z)$ $\wedge \Theta(z, y)$, for all $z \in X$

A reflexive, symmetric, and transitive $L$-fuzzy relation on $X$ is called an $L$-fuzzy equivalence relation on $X$.

\section{L-Fuzzy Congruences of Ockham Algebras}

In this section, we give various characterizations of an $L$-fuzzy congruence relation of an Ockham algebra. Throughout this section and the rest, $A$ stands for an Ockham algebra $(A, f)$.

Definition 9. An $L$-fuzzy equivalence relation $\Theta$ on $A$ is called an $L$-fuzzy congruence relation on $A$ if it satisfies the following conditions:

(1) $\Theta(a \wedge c, b \wedge d) \wedge \Theta(a \vee c, b \vee d) \geq \Theta(a, b) \wedge \Theta(c, d)$

(2) $\Theta(f(a), f(b)) \geq \Theta(a, b)$, for all $a, b, c, d \in A$

An $L$-fuzzy equivalence relation on $A$ that satisfies condition (1) is called a lattice $L$-fuzzy congruence on $A$. The following two lemmas give important characterization for $L$-fuzzy congruence relations in Ockham algebras.

Lemma 1. An L-fuzzy relation $\Theta$ on $A$ is an L-fuzzy congruence relation on $A$ if and only if $\Theta_{\alpha}$ is a congruence relation on $A$, for all $\alpha \in L$.

Lemma 2. An L-fuzzy equivalence relation $\Theta$ on $A$ is an $L$-fuzzy congruence relation on $A$ if and only if, for any $a, b, x \in A$,

$$
\Theta(f(a), f(b)) \wedge \Theta(a \wedge x, b \wedge x) \wedge \Theta(a \vee x, b \vee x) \geq \Theta(a, b)
$$

For any $a \in L$ and $L$-fuzzy congruence relation $\Theta$, define an $L$-fuzzy subset $\Theta_{a}$ of $A$ by

$$
\Theta_{a}(x)=\Theta(a, x), \quad \text { for all } x \in A .
$$

We call $\Theta_{a}$ an $L$-fuzzy congruence class of $\Theta$ determined by $a$, and in particular, $\Theta_{0}$ is called the kernel of $\Theta$. One can easily observe that kernel $\Theta_{0}$ of $\Theta$ is an $L$-fuzzy ideal of $A$.

Lemma 3. Let $\Theta$ be a fuzzy congruence on $A$. For any $x, y \in A$, the following holds

(1) $\Theta_{x}=\Theta_{y}$ if and only if $\Theta(x, y)=1$

(2) Either $\Theta_{x}=\Theta_{y}$ or there exists $\alpha \in L-\{1\}$ such that $\Theta_{x} \cap \Theta_{y} \subseteq \bar{\alpha}$

Let us put $A / \Theta=\{\Theta(x): x \in A\}$ and define binary operations $\wedge, \vee$ and a unary operation $f$ on $A / \Theta$ by

$$
\begin{aligned}
\Theta(x) \wedge \Theta(y) & =\Theta(x \wedge y), \\
\Theta(x) \vee \Theta(y) & =\Theta(x \vee y), \\
f(\Theta(x)) & =\Theta(f(x)) .
\end{aligned}
$$

It is routine to verify that $\left((A / \Theta), \wedge, \vee, f, \Theta_{0}, \Theta_{1}\right)$ is an Ockham algebra, and it is called the quotient Ockham algebra of $A$ modulo $\Theta$. For an $L$-fuzzy subset $\mu$ of $A$, we write $\Theta_{L}(\mu)$ (respectively, $\left.\left(\Theta_{\text {lat }}\right)_{L}(\mu)\right)$ to denote the smallest $L$-fuzzy congruence (respectively lattice $L$-fuzzy congruence) on $A$ containing $\mu \times \mu$. It was proved in [34] that, for any $x, y \in A$,

$$
\left(\Theta_{\text {lat }}\right)_{L}(\mu)(x, y)=\vee\{\mu(i): x \vee i=y \vee i\},
$$

whenever $\mu$ is an $L$-fuzzy ideal of $A$ and

$$
\left(\Theta_{\text {lat }}\right)_{L}(\mu)(x, y)=\bigvee\{\mu(j): x \wedge j=y \wedge j\},
$$

whenever $\mu$ is an $L$-fuzzy filter of $A$.

For a given $L$-fuzzy ideal $\mu$ of $A$, we shall now investigate the smallest $L$-fuzzy congruence $\Theta_{L}(\mu)$ of $A$ containing $\mu \times \mu$.

Definition 10. An $L$-fuzzy subset $\mu$ of $A$ is called an $L$-fuzzy down set (respectively, $L$-fuzzy up set) if, for any $x, y \in A$, $\mu(x) \geq \mu(y)$ (respectively, $\mu(x) \leq \mu(y)$ ) whenever $x \leq y$.

Lemma 4. Let $\mu$ be an L-fuzzy subset of A. Then, the L-fuzzy subset $\mu^{\downarrow}$ of $A$ defined by

$$
\mu^{\downarrow}(x)=\bigvee\{\mu(y): x \leq y\}, \quad \text { for all } x \in A,
$$

is the smallest L-fuzzy down set containing $\mu$.

Proof. Let $x \in A$. Since $x \leq x$, we clearly have

$$
\mu^{\downarrow}(x)=\bigvee\{\mu(y): x \leq y\} \geq \mu(x) .
$$

Thus, $\mu \subseteq \mu^{\downarrow}$. Let $x, y \in A$ such that $x \leq y$. Now,

$$
\begin{aligned}
\mu^{\downarrow}(x) & =\bigvee\{\mu(z): x \leq z\} \\
& \geq \bigvee\{\mu(z): y \leq z\} \\
& =\mu^{\downarrow}(y) .
\end{aligned}
$$

Therefore, $\mu^{\downarrow}$ is an $L$-fuzzy down set of $A$. Let $\sigma$ be any $L$-fuzzy down set of $A$ such that $\mu \subseteq \sigma$. For any $x \in A$, we have

$$
\mu^{\downarrow}(x)=\bigvee\{\mu(y): x \leq y\} \leq \bigvee\{\sigma(y): x \leq y\}=\sigma(x) .
$$

This implies that $\mu^{\downarrow} \subseteq \sigma$. Therefore, $\mu^{\downarrow}$ is the smallest $L$-fuzzy down set of $A$ containing $\mu$.

Dually, we have the following lemma.

Lemma 5. Let $\mu$ be an L-fuzzy subset of A. Then, the L-fuzzy subset $\mu^{\uparrow}$ of $A$ defined by

$$
\mu^{\uparrow}(x)=\vee\{\mu(y): y \leq x\}, \quad \text { for all } x \in A,
$$

is the smallest L-fuzzy up set containing $\mu$.

Lemma 6. Let $\mu$ be an L-fuzzy ideal of A. For each nonnegative integer $n$, define $\mu_{2 n}=\left(f^{2 n}(\mu)\right)^{\downarrow}, \mu^{2 n+1}=\left(f^{2 n+1}(\mu)\right)^{\uparrow}$. Then, $\mu_{2 n}$ is an L-fuzzy ideal and $\mu^{2 n+1}$ is an L-fuzzy filter of $A$.

Proof. Now, since 


$$
\begin{aligned}
\mu_{2 n}(0) & =\left(f^{2 n}(\mu)\right)^{\downarrow}(0) \\
& =\bigvee\left\{f^{2 n}(\mu)(y): 0 \leq y\right\} \\
& \geq f^{2 n}(\mu)(0) \quad(\text { Since } 0 \leq 0) \\
& =\vee\left\{\mu(a): a \in\left(f^{2 n}\right)^{-1}(0)\right\} \quad\left(\text { Since }\left(f^{2 n}\right)^{-1}(0) \neq \varnothing\right) \\
& \geq \mu(0)=1 \quad\left(\text { Since } 0 \in\left(f^{2 n}\right)^{-1}(0)\right) .
\end{aligned}
$$

We have $\mu_{2 n}(0)=1$.

Again, let $x, y \in A$. Then, if $(\forall w)(x \leq w),\left(f^{2 n}\right)^{-1}(w)=$ $\varnothing$ or $(\forall z)(y \leq z),\left(f^{2 n}\right)^{-1}(z)=\varnothing$, then we have

$$
\begin{aligned}
\mu_{2 n}(x) \wedge \mu_{2 n}(y) & =\left(f^{2 n}(\mu)\right)^{\downarrow}(x) \wedge\left(f^{2 n}(\mu)\right)^{\downarrow}(y) \\
& =\vee\left\{f^{2 n}(\mu)(w): x \leq w\right\} \wedge \vee\left\{f^{2 n}(\mu)(z): y \leq z\right\} \\
& =0 \\
& \leq \mu_{2 n}(x \vee y) .
\end{aligned}
$$

If $\quad(\exists w)(x \leq w), \quad\left(f^{2 n}\right)^{-1}(w) \neq \varnothing \quad$ and $\quad(\exists z)(y \leq z)$, $\left(f^{2 n}\right)^{-1}(z) \neq \varnothing$, we have

$$
\begin{aligned}
\mu_{2 n}(x) \wedge \mu_{2 n}(y)= & \left(f^{2 n}(\mu)\right)^{\downarrow}(x) \wedge\left(f^{2 n}(\mu)\right)^{\downarrow}(y) \\
= & \vee\left\{f^{2 n}(\mu)(w): x \leq w\right\} \wedge \sup \left\{f^{2 n}(\mu)(z): y \leq z\right\} \\
= & \vee\left\{\mu(a): x \leq f^{2 n}(a), \quad a \in A\right\} \wedge \\
& \vee\left\{\mu(b): y \leq f^{2 n}(b), \quad b \in A\right\} \\
= & \vee\left\{\mu(a) \wedge \mu(b): x \leq f^{2 n}(a), y \leq f^{2 n}(b) \quad a, b \in A\right\} \\
\leq & \vee\left\{\mu(a \vee b): x \vee y \leq f^{2 n}(a \vee b), \quad a, b \in A\right\} \\
\leq & \left(f^{2 n}(\mu)\right)^{\downarrow}(x \vee y) \\
= & \mu_{2 n}(x \vee y) .
\end{aligned}
$$

Again, as $\mu_{2 n}$ is an $L$-fuzzy down set, we clearly have $\mu_{2 n}(x) \wedge \mu_{2 n}(y) \geq \mu_{2 n}(x \vee y)$, and hence, $\mu_{2 n}(x \vee y)=\mu_{2 n}$ $(x) \wedge \mu_{2 n}(y)$. Therefore, $\mu_{2 n}$ is an $L$-fuzzy ideal of $A$. Analogously, we can prove that $\mu^{2 n+1}$ is an $L$-fuzzy filter of A.

Lemma 7. Let $\mu$ be any L-fuzzy ideal of A. Put $\mu_{\mathrm{oo}}=\vee_{n \geq 0} \mu_{2 n}$ and $\mu^{\circ}=\mathrm{V}_{n \geq 0} \mu^{2 n+1}$. Then,

$$
\begin{aligned}
& \mu^{\circ}(f(x)) \geq \mu_{\circ}(x), \quad \text { for all } x \in A . \\
& \mu_{\circ}(f(x)) \geq \mu^{\circ}(x),
\end{aligned}
$$

Proof. Let $x \in A$. If $(\forall y)(x \leq y),\left(f^{2 n}\right)^{-1}(y)=\varnothing$, we have

$$
\begin{aligned}
\mu_{\circ}(x) & =\left(\bigvee_{n \geq 0} \mu_{2 n}\right)(x)=\bigvee_{n \geq 0}\left(\bigwedge_{n \geq 0} \mu_{2 n}(x)\right) \\
& =\bigvee_{n \geq 0}\left(\bigwedge_{n \geq 0}\left(f^{2 n}(\mu)\right)^{\downarrow}(x)\right) \\
& =\bigvee_{n \geq 0} \bigwedge_{n \geq 0}\left\{\vee\left\{f^{2 n}(\mu)(y): x \leq y\right\}\right. \\
& =0 \leq \mu^{\circ}(f(x)) .
\end{aligned}
$$

If $(\exists y)(x \leq y),\left(f^{2 n}\right)^{-1}(y) \neq \varnothing$, then

$$
\begin{aligned}
& \mu_{\mathrm{o}}(x)=\left(\underset{n \geq 0}{\vee} \mu_{2 n}\right)(x) \\
& =\bigvee_{n \geq 0} \bigwedge_{n \geq 0} \mu_{2 n}(x) \\
& =\underset{n \geq 0}{ } \wedge \wedge_{n \geq 0}\left(f^{2 n}(\mu)\right)^{\downarrow}(x) \\
& =\bigvee_{n \geq 0} \wedge \wedge_{n \geq 0}\left(\vee\left\{f^{2 n}(\mu)(y): x \leq y\right\}\right) \\
& \left.=\bigvee_{n \geq 0} \wedge\left\{\vee\left\{\vee \vee \mu(z): f^{2 n}(z)=y\right\}: x \leq y\right\}\right\} \\
& \left.\leq \vee{ }_{n \geq 0} \wedge\left\{0\left\{\vee \vee \mu(z): f^{2 n+1}(z)=f(y)\right\}: f(y) \leq f(x)\right\}\right\} \\
& =\bigvee_{n \geq 0} \wedge\left\{\vee\left\{f^{2 n+1}(\mu)(f(y)): f(y) \leq f(x)\right\}\right\} \\
& \leq \underset{n \geq 0}{\vee} \wedge{ }_{n \geq 0}\left(f^{2 n+1}(\mu)\right)^{\uparrow}(f(x)) \\
& =\bigvee_{n \geq 0} \underset{n \geq 0}{\wedge} \mu^{2 n+1}(f(x))=\left(\bigvee_{n \geq 0} \mu^{2 n+1}\right)(f(x)) \\
& =\mu^{\circ}(f(x)) \text {. }
\end{aligned}
$$

Similarly, we can show that $\mu_{\mathrm{oo}}(f(x)) \geq \mu^{\circ}(x)$.

Lemma 8. Let $\mu$ be an L-fuzzy ideal of A. Then, the L-fuzzy binary relation $\Omega_{L}$ on $A$ defined by

$$
\Omega_{L}(\mu)(x, y)=\vee\left\{\mu_{\mathrm{oo}}(a) \wedge \mu^{\circ}(b):(x \vee a) \wedge b=(y \vee a) \wedge b\right\} \text {, }
$$

for all $x, y \in A$ is an L-fuzzy congruence relation on $A$.

Proof. Clearly, $\Omega_{L}(\mu)$ is reflexive and symmetric. We show that it is transitive. Let $x, y, z \in A$. If $(x \vee a) \wedge b=(y \vee a)$ $\wedge b,(y \vee c) \wedge d=(z \vee c) \wedge d$, then it can be easily verified that $(x \vee(a \vee c)) \wedge(b \wedge d)=(z \vee(a \vee c)) \wedge(b \wedge d)$. Thus, we have 


$$
\begin{aligned}
\Omega_{L}(\mu)(x, y) \wedge \Omega_{L}(\mu)(y, z)= & \vee\left\{\mu_{\circ}(a) \wedge \mu^{\circ}(b):(x \vee a) \wedge b=(y \vee a) \wedge b\right\} \\
& \wedge \vee\left\{\mu_{\circ}(c) \wedge \mu^{\circ}(d):(y \vee c) \wedge d=(z \vee c) \wedge d\right\} \\
= & \vee\left\{\mu_{\circ \circ}(a) \wedge \mu_{\circ}(c) \wedge \mu^{\circ}(b) \wedge \mu^{\circ}(d):(x \vee a) \wedge b=(y \vee a) \wedge b,(y \vee c) \wedge d=(z \vee c) \wedge d\right\} \\
\leq & \vee\left\{\mu_{\circ}(a \vee c) \wedge \mu^{\circ}(b \wedge d):(x \vee(a \vee c)) \wedge(b \wedge d)=(z \vee(a \vee c)) \wedge(b \wedge d)\right\} \\
= & \Omega_{L}(\mu)(x, z) .
\end{aligned}
$$

Therefore, $\Omega_{L}(\mu)$ is transitive, and hence, it is an $L$ - fuzzy equivalence relation on $A$.

Next, we show that $\Omega_{L}(\mu)$ satisfies the substitution properties. $(x \vee a) \wedge b=(y \vee a) \wedge b,(z \vee c) \wedge d=(w \vee c) \wedge d$, then, after routine work, we obtain

$$
\begin{aligned}
((x \wedge z) \vee(a \vee c)) \wedge(b \wedge d)= & ((y \wedge w) \vee(a \vee c)) \wedge(b \wedge d) \\
\Omega_{L}(\mu)(x, y) \wedge \Omega_{L}(\mu)(z, w)= & \vee\left\{\mu_{\circ}(a) \wedge \mu^{\circ}(b):(x \vee a) \wedge b=(y \vee a) \wedge b\right\} \wedge \\
& \vee\left\{\mu_{\circ}(c) \wedge \mu^{\circ}(d):(z \vee c) \wedge d=(w \vee c) \wedge d\right\} \\
= & \vee\left\{\mu_{\circ \circ}(a) \wedge \mu_{\circ}(c) \wedge \mu^{\circ}(b) \wedge \mu^{\circ}(d):(x \vee a) \wedge b=(y \vee a) \wedge b,(z \vee c) \wedge d=(w \vee c) \wedge d\right\} \\
\leq & \vee\left\{\mu_{\circ \circ}(a \vee c) \wedge \mu^{\circ}(b \wedge d):((x \wedge z) \vee(a \vee c)) \wedge(b \wedge d)=((y \wedge w) \vee(a \vee c)) \wedge(b \wedge d)\right\} \\
= & \Omega_{L}(\mu)(x \wedge z, y \wedge w) .
\end{aligned}
$$

Similarly, we can show that

$\Omega_{L}(\mu)(x, y) \wedge \Omega_{L}(\mu)(z, w) \leq \Omega_{L}(\mu)(x \vee z, y \vee w)$.

Finally, if $(x \vee a) \wedge b=(y \vee a) \wedge b$, then it can be easily verified

$$
\begin{aligned}
& (f(x) \vee f(b)) \wedge(f(a) \vee f(b)) \\
& \quad=(f(y) \vee f(b)) \wedge(f(a) \vee f(b)) .
\end{aligned}
$$

Now,

$$
\begin{aligned}
\Omega_{L}(\mu)(x, y)= & \vee\left\{\mu_{\circ}(a) \wedge \mu^{\circ}(b):(x \vee a) \wedge b=(y \vee a) \wedge b\right\} \\
\leq & \vee\left\{\mu_{\circ \circ}(f(b)) \wedge \mu^{\circ}(f(a)):[f(x) \vee f(b)] \wedge\right. \\
& {[f(a) \vee f(b)]=[f(y) \vee f(b)] \wedge[f(a) \vee f(b)]\} } \\
\leq & \vee\left\{\mu_{\circ}(f(b)) \wedge \mu^{\circ}(f(a) \vee f(b)):[f(x) \vee f(b)] \wedge[f(a)\right. \\
& \vee f(b)]=[f(y) \vee f(b)] \wedge[f(a) \vee f(b)], \quad a, b \in A\} \\
= & \Omega_{L}(\mu)(f(x), f(y)) .
\end{aligned}
$$

Thus, $\Omega_{L}(\mu)$ is an $L$-fuzzy congruence on $A$.

Theorem 1. For any L-fuzzy ideal $\mu$ of $A$,

$$
\Omega_{L}(\mu)=\left(\Theta_{\text {lat }}\right)_{L}\left(\mu_{\mathrm{oo}}\right) \vee\left(\Theta_{\text {lat }}\right)_{L}\left(\mu^{\circ}\right),
$$

where the join is taken in the lattice of L-fuzzy congruences on $A$.

Proof. We first show that $\left(\Theta_{\text {lat }}\right)_{L}\left(\mu_{\circ \circ}\right) \subseteq \Omega_{L}(\mu)$ and $\left(\Theta_{\text {lat }}\right)_{L}$ $\left(\mu^{\circ}\right) \subseteq \Omega_{L}(\mu)$. Now, for any $x, y \in A$, we have

$$
\begin{aligned}
& \left(\left(\Theta_{\text {lat }}\right)_{L}\left(\mu_{\mathrm{oo}}\right)(x, y)\right)=\vee\left\{\mu_{\mathrm{oo}}(s): x \vee s=y \vee s\right\} \\
& \leq \vee\left\{\mu_{\mathrm{oo}}(s) \wedge \mu^{\circ}(f(s)):(x \vee s) \wedge f(s)=(y \vee s) \wedge f(s)\right\} \\
& \leq \Omega_{L}(\mu)(x, y) .
\end{aligned}
$$

Hence, $\left(\Theta_{\text {lat }}\right)_{L}\left(\mu_{\circ \circ}\right) \subseteq \Omega_{L}(\mu)$. Similarly, we have $\left(\Theta_{\text {lat }}\right)_{L}\left(\mu^{\circ}\right) \subseteq \Omega_{L}(\mu)$. This implies that

$$
\left(\Theta_{\text {lat }}\right)_{L}\left(\mu_{\mathrm{oo}}\right) \vee\left(\Theta_{\text {lat }}\right)_{L}\left(\mu^{\circ}\right) \subseteq \Omega_{L}(\mu) .
$$

To establish the reverse inclusion, let $x, y \in A$. Then, 


$$
\begin{aligned}
\Omega_{L}(\mu)(x, y) & =\vee\left\{\mu_{\mathrm{o}}(s) \wedge \mu^{\circ}(t):(x \vee s) \wedge t=(y \vee s) \wedge t\right\} \\
& \leq \vee\left\{\mu_{\mathrm{o}}(s) \wedge \mu^{\circ}(t): x \wedge(x \vee s) \wedge t=x \wedge(y \vee s) \wedge t\right\} \\
& \leq \vee\left\{\mu^{\circ}(t): x \wedge t=x \wedge(y \vee s) \wedge t\right\} \\
& =\left(\Theta_{\text {lat }}\right)_{L}\left(\mu^{\circ}\right)(x, x \wedge(y \vee s)), \\
\Omega_{L}(\mu)(x, y) & =\vee\left\{\mu_{\mathrm{oo}}(s) \wedge \mu^{\circ}(t):(x \vee s) \wedge t=(y \vee s) \wedge t\right\} \\
& \leq \vee\left\{\mu_{\mathrm{oo}}(s) \wedge \mu^{\circ}(t): x \wedge(x \vee s) \wedge t=x \wedge(y \vee s) \wedge t\right\} \\
& =\vee\left\{\mu_{\mathrm{oo}}(s) \wedge \mu^{\circ}(t): x \wedge t=x \wedge(y \vee s) \wedge t\right\} \\
& \leq \vee\left\{\mu_{\mathrm{oo}}(s) \wedge \mu^{\circ}(t):(x \wedge t) \vee s=(x \wedge(y \vee s) \wedge t) \vee s\right\} \\
& \leq \vee\left\{\mu_{\mathrm{oo}}(s):(x \wedge(y \vee s)) \vee s=(x \wedge y) \vee s\right\} \\
& =\left(\Theta_{\mathrm{lat}}\right)_{L}\left(\mu_{\mathrm{oo}}\right)(x \wedge(y \vee s), x \wedge y) .
\end{aligned}
$$

This implies that

$$
\begin{aligned}
\Omega_{L}(\mu)(x, y) \leq & \left(\Theta_{\text {lat }}\right)_{L}\left(\mu^{\circ}\right)(x, x \wedge(y \vee s)) \\
& \wedge\left(\Theta_{\text {lat }}\right)_{L}\left(\mu_{\mathrm{o}}\right)(x \wedge(y \vee s), x \wedge y) \\
\leq & \left(\left(\Theta_{\text {lat }}\right)_{L}\left(\mu^{\circ}\right) \vee\left(\Theta_{\text {lat }}\right)_{L}\left(\mu_{\circ \circ}\right)\right)(x, x \wedge y) .
\end{aligned}
$$

Similarly,

$\Omega_{L}(\mu)(x, y) \leq\left(\left(\Theta_{\text {lat }}\right)_{L}\left(\mu^{\circ}\right) \vee\left(\Theta_{\text {lat }}\right)_{L}\left(\mu_{\circ}\right)\right)(x \wedge y, y)$. Again,

$$
\begin{aligned}
\Omega_{L}(\mu)(x, y) \leq & \left(\left(\Theta_{\text {lat }}\right)_{L}\left(\mu^{\circ}\right) \vee\left(\Theta_{\text {lat }}\right)_{L}\left(\mu_{\mathrm{oo}}\right)\right)(x, x \wedge y) \\
& \vee\left(\left(\Theta_{\text {lat }}\right)_{L}\left(\mu^{\circ}\right) \vee\left(\Theta_{\text {lat }}\right)_{L}\left(\mu_{\circ}\right)\right)(x \wedge y, y) \\
\leq & \left(\left(\Theta_{\text {lat }}\right)_{L}\left(\mu^{\circ}\right) \vee\left(\Theta_{\text {lat }}\right)_{L}\left(\mu_{\mathrm{o}}\right)\right)(x, y) .
\end{aligned}
$$

Hence, $\Omega_{L}(\mu)=\left(\left(\Theta_{\text {lat }}\right)_{L}\left(\mu^{\circ}\right) \vee\left(\Theta_{\text {lat }}\right)_{L}\left(\mu_{\text {oo }}\right)\right)$.

Corollary 1. $\Omega_{L}(\mu)$ is the smallest L-fuzzy congruence on $A$ containing $\mu \times \mu$.

\section{L-Fuzzy Kernel Ideals}

In this section, we study $L$-fuzzy kernel ideals in Ockham algebras and give several characterizations for them.

Definition 11. An $L$-fuzzy ideal $\mu$ on $A$ is called a kernel $L$-fuzzy ideal if $\mu=\Theta_{0}$ for some $L$-fuzzy congruence $\Theta$ of $A$.

The following observation is immediate.

Lemma 9. If $\mu$ is an L-fuzzy ideal of A such that $f^{2}(\mu) \subseteq \mu$, then, for every $n$,

$$
\begin{gathered}
f^{2 n}(\mu) \subseteq \mu, \\
f^{2 n+1}(\mu) \subseteq(f(\mu))^{\uparrow},
\end{gathered}
$$

from which it follows that

$$
\begin{aligned}
\mu_{\mathrm{o}} & =\mu, \\
\mu^{\circ} & =(f(\mu))^{\uparrow} .
\end{aligned}
$$

The following lemma gives an internal characterization for $L$-fuzzy kernel ideals in Ockaham algebras.

Lemma 10. An L-fuzzy ideal $\mu$ of $A$ is an L-fuzzy kernel ideal if and only if it satisfies the following properties:

(1) $f^{2}(\mu) \subseteq \mu$

(2) $(f(\mu))^{\uparrow}(t) \wedge \mu(x \wedge t) \leq \mu(x)$, for all $x, t \in A$

Proof. Let $\mu$ be an $L$-fuzzy kernel ideal of $A$. Then, there exists an $L$-fuzzy congruence $\Theta$ on $A$ such that $\mu=\Theta_{0}$. Let $x \in A$. If $\left(\left(f^{2}\right)^{-1}(x)\right)=\varnothing$, then $f^{2}(\mu)(x)=0 \leq \mu(x)$. Let $\left(f^{2}(x)\right)^{-1} \neq \varnothing$, and consider the following:

$$
\begin{aligned}
f^{2}(\mu)(x)= & \bigvee\left\{\mu(a): x=f^{2}(a)\right\} \\
= & \bigvee\left\{\Theta(a, 0): x=f^{2}(a)\right\} \\
\leq & \Theta\left(f^{2}(a), 0\right) \\
= & \Theta(x, 0) \\
& \cdot \mu(x) .
\end{aligned}
$$

Hence, $f^{2}(\mu)(x) \subseteq \mu(x)$, for all $x \in A$. Therefore, (1) holds. To prove (2), let $x, t \in A$. If $\left(f^{-1}(t)\right)=\varnothing$, clearly (2) holds. Suppose that $f^{-1}(t) \neq \varnothing$. Then,

$$
\begin{aligned}
(f(\mu))^{\uparrow}(t) \wedge \mu(x \wedge t) & =\bigvee\{f(\mu)(a)): a \leq t\} \wedge \mu(x \wedge t) \\
& =\bigvee\{\vee\{\mu(y): f(y)=a\}: a \leq t\} \wedge \mu(x \wedge t) \\
& =\bigvee\{\mu(y): f(y) \leq t\} \wedge \mu(x \wedge t) \\
& =\vee\{\Theta(y, 0): f(y) \leq t\} \wedge \Theta(t \wedge x, 0) \\
& \leq \Theta(f(y), 1) \wedge \Theta(t \wedge x, 0) \\
& \leq \Theta(t, 1) \wedge \Theta(t \wedge x, 0) \\
& \leq \Theta(t \wedge x, x) \wedge \Theta(t \wedge x, 0) \\
& \leq \Theta(x, 0) \\
& =\mu(x) .
\end{aligned}
$$

Conversely, suppose that $\mu$ is an $L$-fuzzy ideal of $A$ satisfying conditions (1) and (2). Consider the $L$-fuzzy congruence $\Omega_{L}(\mu)$ given in Lemma 8 . Now, we claim that $\mu=\left(\Omega_{L}(\mu)\right)_{0}$. Let $x \in A$. Then, 
$\mu(x)=\mu(x) \wedge \mu(0)=(\mu \times \mu)(x, 0) \subseteq \Omega_{L}(\mu)(x, 0)=\left(\Omega_{L}(\mu)\right)_{0}(x)$,

and hence, $\mu \subseteq\left(\Omega_{L}(\mu)\right)_{0}$. To show the other inclusion,

$$
\begin{aligned}
\left(\Omega_{L}(\mu)\right)_{0}(x)= & \Omega_{L}(\mu)(x, 0) \\
= & \vee\left\{\mu_{\circ}(s) \wedge \mu^{\circ}(t):(x \vee s) \wedge t=(0 \vee s) \wedge t\right\} \\
= & \vee\left\{\mu(s) \wedge(f(\mu))^{\uparrow}(t):\right. \\
& (x \vee s) \wedge t=s \wedge t\}(\text { by Lemma 9) } \\
\leq & \vee\left\{\mu(s \wedge t) \wedge(f(\mu))^{\uparrow}(t):(x \vee s) \wedge t=s \wedge t\right\} \\
= & \vee\left\{\mu((x \vee s) \wedge t) \wedge(f(\mu))^{\uparrow}(t):(x \vee s) \wedge t=s \wedge t\right\} \\
\leq & \mu(x \vee s) \\
\leq & \mu(x) .
\end{aligned}
$$

Hence, $\left(\Omega_{L}(\mu)\right)_{0} \subseteq \mu$. Therefore, $\left(\Omega_{L}(\mu)\right)_{0}=\mu$.

Corollary 2. If $\mu$ is a proper kernel L-fuzzy ideal of $A$, then

$$
\mu \cap f(\mu) \subseteq \bar{\alpha}
$$

for some $\alpha \in L-\{1\}$.

Proof. Suppose that $\mu$ is a proper kernel $L$-fuzzy ideal of $A$. Then, $\mu=\Theta_{0}$, for some $L$-fuzzy congruence $\Theta$ of $A$ and $\mu(a) \neq 1$, for some $a \in A$. Put $\alpha=\mu(a)$. Now, we claim that $\mu \cap f(\mu) \subseteq \bar{\alpha}$. Let $x \in A$. Then, the claim clearly holds if $f^{-1}(x)=\varnothing$. Suppose that $f^{-1}(x) \neq \varnothing$. Then,

$$
\begin{aligned}
(\mu \cap f(\mu))(x) & =\mu(x) \wedge f(\mu)(x) \\
& =\mu(x) \wedge \vee\{\mu(y): x=f(y)\} \\
& =\Theta_{0}(x) \wedge \vee\left\{\Theta_{0}(y): x=f(y)\right\} \\
& =\Theta(x, 0) \wedge \vee\{\Theta(y, 0): x=f(y)\} \\
& \leq \Theta(x, 0) \wedge \Theta(x, 1) \\
& \leq \Theta(1,0)=\mu(1) \leq \mu(a)=\alpha .
\end{aligned}
$$

Hence, the claim is true.

Lemma 11. Arbitrary intersection of a family of kernel L-fuzzy ideals of A is a kernel L-fuzzy ideal.

We denote the set of all kernel $L$-fuzzy ideals of $A$ by $\mathscr{F} \mathscr{I}_{K}(A)$.

Theorem 2. The set $\mathscr{F} \mathscr{I}_{K}(A)$ of kernels L-fuzzy ideal of $A$ ordered by set inclusion forms a complete lattice.
Consider the subset $\mathscr{F} \mathscr{I}_{2}(A)=\left\{\mu \in \mathscr{F} \mathscr{I}(A): f^{2}(\mu)\right.$ $\subseteq \mu\}$. Then, we have the following results.

\section{Theorem 3.}

(1) $\mathscr{F} \mathscr{I}_{2}(A)$ is a complete sublattice of $\mathscr{F} \mathscr{I}(A)$

(2) If $\mu$ is a kernel L-fuzzy ideal of $A$, then $\mu \in \mathscr{F}_{\mathscr{I}_{2}}(A)$

Proof. Let $\left\{\mu_{i}: i \in \Delta\right\}$ be a nonempty subset of $\mathscr{F}_{2} \mathscr{I}_{2}(A)$. Then, $f^{2}\left(\mu_{i}\right) \subseteq \mu_{i}$, for all $i \in \Delta$. Now, we claim that $\cap_{i \in \Delta} \mu_{i} \in \mathscr{F} \mathscr{I}_{2}(A)$.

Let $x \in A$. Suppose that $\left(f^{2}\right)^{-1}(x)=\varnothing$. Then,

$$
f^{2}\left(\bigcap_{i \in \Delta} \mu_{i}\right)(x)=0 \leq\left(\bigcap_{i \in \Delta} \mu_{i}\right)(x) .
$$

Now, suppose that $\left(f^{2}\right)^{-1}(x) \neq \varnothing$. Then,

$$
\begin{aligned}
f^{2}\left(\bigcap_{i \in \Delta} \mu_{i}\right)(x) & =\sup \left\{\left(\bigcap_{i \in \Delta} \mu_{i}\right)(a): x=f^{2}(a)\right\} \\
& =\sup \left\{\inf _{i \in \Delta} \mu_{i}(a): x=f^{2}(a)\right\} \\
& =\inf _{i \in \Delta}\left\{\sup \mu_{i}(a): x=f^{2}(a)\right\} \\
& =\inf _{i \in \Delta} f^{2}\left(\mu_{i}\right)(x) \\
& \leq \inf _{i \in \Delta} \mu_{i}(x) \\
& =\left(\bigcap_{i \in \Delta} \mu_{i}\right)(x) .
\end{aligned}
$$

Hence, the claim is true. Since $\chi_{A}$ is greatest element of $\mathscr{F} \mathscr{I}_{2}(A)$, we have $\mathscr{F} \mathscr{I}_{2}(A)$ is a complete sublattice of $\mathscr{F} \mathscr{I}(A)$

It follows from Lemma 10.

Lemma 12. Let $\mu$ be an L-fuzzy ideal of A. Then, the L-fuzzy subset $\tilde{\mu}$ of $A$ defined by

$$
\widetilde{\mu}(x)=\bigvee\{\mu(i) \wedge \mu(x \wedge f(i)): i \in A\}, \quad \text { for all } x \in A,
$$

is an L-fuzzy ideal of A containing $\mu$.

Proof. Suppose that $\mu$ be an $L$-fuzzy ideal of $A$. Now, since

$$
\begin{aligned}
\widetilde{\mu}(0) & =\bigvee\{\mu(i) \wedge \mu(0 \wedge f(i)): i \in A\} \\
& =\bigvee\{\mu(i): i \in A\} \\
& \geq \mu(0)=1 .
\end{aligned}
$$

We have $\tilde{\mu}(0)=1$. Let $x, y \in A$. Then, 


$$
\begin{aligned}
\widetilde{\mu}(x) \wedge \widetilde{\mu}(y)= & \vee\{\mu(i) \wedge \mu(x \wedge f(i)): \quad i \in A\} \\
& \wedge \vee\{\mu(j) \wedge \mu(y \wedge f(j)): \quad j \in A\} \\
= & \vee\{\mu(i) \wedge \mu(x \wedge f(i)) \wedge \mu(j) \wedge \mu(y \wedge f(j)): \quad i, j \in A\} \\
\leq & \vee\{\mu(i \vee j) \wedge \mu(x \wedge f(i \vee j)) \wedge \mu(y \wedge f(i \vee j)): \quad i, j \in A\} \\
\leq & \vee\{\mu(i \vee j) \wedge \mu((x \vee y) \wedge f(i \vee j)): \quad i \vee j \in A\} \\
& =\widetilde{\mu}(x \vee y) .
\end{aligned}
$$

Again since

$$
\begin{aligned}
\widetilde{\mu}(x) & =\vee\{\mu(i) \wedge \mu(x \wedge f(i)): \quad i \in A\} \\
& \geq \vee\{\mu(i) \wedge \mu((x \vee y) \wedge f(i)): \quad i \in A\} \\
& =\widetilde{\mu}(x \vee y) .
\end{aligned}
$$

Similarly, we have $\widetilde{\mu}(y) \geq \widetilde{\mu}(x \vee y)$, and hence, $\widetilde{\mu}(x) \wedge$ $\widetilde{\mu}(y) \geq \widetilde{\mu}(x \vee y)$. Therefore, $\widetilde{\mu}(x \vee y)=\widetilde{\mu}(x) \wedge \widetilde{\mu}(y)$. Again, since, for any $x \in A$,

$$
\begin{aligned}
\widetilde{\mu}(x) & =\vee\{\mu(i) \wedge \mu(x \wedge f(i)): \quad i \in A\} \\
& \geq \mu(0) \wedge \mu(x \wedge f(0)) \\
& =1 \wedge \mu(x \wedge 1) \\
& =\mu(x),
\end{aligned}
$$

we have $\mu \subseteq \tilde{\mu}$. Therefore, $\widetilde{\mu}$ is an $L$-fuzzy ideal of $A$ containing $\mu$.

In the following theorem, we give a necessary and sufficient condition for an $L$-fuzzy ideal of an Ockham algebra to be a kernel $L$-fuzzy ideal.

Theorem 4. An L-fuzzy ideal $\mu$ of $A$ is a kernel L-fuzzy ideal of $A$ if and only if $f^{2}(\mu) \subseteq \mu$ and $\mu=\widetilde{\mu}$.

Proof. Suppose that $\mu$ is a kernel $L$-fuzzy ideal of $A$. Then, by Lemmas 10 and 12 , we have $f^{2}(\mu) \subseteq \mu$ and $\mu \subseteq \widetilde{\mu}$. Now, since, for any $x \in A$,

$$
\begin{aligned}
\widetilde{\mu}(x) & =\bigvee\{\mu(i) \wedge \mu(x \wedge f(i)): \quad i \in A\} \\
& \leq \vee\{f(\mu)(f(i)) \wedge \mu(x \wedge f(i)): \quad i \in A\} \\
& \leq \vee\left\{f(\mu)^{\uparrow}(f(i)) \wedge \mu(x \wedge f(i)): \in A\right\} \\
& \leq \mu(x),
\end{aligned}
$$

we have $\widetilde{\mu} \subseteq \mu$. Hence, $f^{2}(\mu) \subseteq \mu$ and $\mu=\widetilde{\mu}$.

Conversely, suppose that $\mu$ is an $L$-fuzzy ideal of $A$ satisfying the given condition. Then, by Lemma 11, it is enough to show that

$$
(f(\mu))^{\uparrow}(t) \wedge \mu(x \wedge t) \leq \mu(x), \quad \text { for all } x, t \in A .
$$

Let $x, t \in A$ if $\left(\left(f^{2}\right)^{-1}(t)\right)=\varnothing$; clearly, (57) holds. Suppose that $\left(\left(f^{2}\right)^{-1}(t)\right) \neq \varnothing$. Then,

$$
\begin{aligned}
(f(\mu))^{\uparrow}(t) \wedge \mu(x \wedge t)= & \bigvee\{f(\mu)(a): a \leq t\} \wedge \mu(x \wedge t) \\
= & \vee\{\vee\{\mu(y): f(y)=a\}: a \leq t\} \\
& \wedge \mu(x \wedge t) \\
= & \vee\{\mu(y): f(y) \leq t\} \wedge \mu(x \wedge t) \\
= & \vee\{\mu(y) \wedge \mu(x \wedge t): f(y) \leq t\} \\
\leq & \vee\{\mu(y) \wedge \mu(x \wedge f(y)): f(y) \leq t\} \\
\leq & \widetilde{\mu}(x)=\mu(x) .
\end{aligned}
$$

Hence, $\mu$ is a kernel $L$-fuzzy ideal of $A$.

Theorem 5. Let $\mu$ be an L-fuzzy ideal of A. Then,

(1) If $\mu \in \mathscr{F} \mathscr{I}_{2}(A)$, then $\tilde{\mu} \in F I_{2}(A)$

(2) The mapping $\xi: \mathscr{F}_{2}(A) \longrightarrow \mathscr{F}_{2} \mathscr{I}_{2}(A)$ defined by $\xi(\mu)=\widetilde{\mu}$ is a closure on $\mathscr{F}_{2}(L)$ and $\operatorname{Im} \xi=\mathscr{F} \mathscr{I}_{K}(A)$

Proof. Let $\mu \in \mathscr{F} \mathscr{I}_{2}(A)$. Then, $f^{2}(\mu) \subseteq \mu$. Let $x \in A$. Then, if $\left(\left(f^{2}\right)^{-1}(x)\right)=\varnothing$, obviously, $\widetilde{\mu} \in \mathscr{F}_{2}(A)$. Suppose that $\left(\left(f^{2}\right)^{-1}(x)\right)=\varnothing$. Then,

$$
\begin{aligned}
f^{2}(\widetilde{\mu})(x)= & \vee\left\{(\widetilde{\mu})(y): x=f^{2}(y)\right\} \\
= & \vee\left\{\vee\{\mu(i) \wedge \mu(y \wedge f(i)): \quad i \in A\}: x=f^{2}(y)\right\} \\
= & \vee\left\{\mu(i) \wedge \mu(y \wedge f(i)): x=f^{2}(y), \quad i \in A\right\} \\
\leq & \vee\left\{f^{2}(\mu)\left(f^{2}(i)\right) \wedge f^{2}(\mu)\left(f^{2}\right)\right. \\
& \left.(y \wedge f(i)): x=f^{2}(y), \quad i \in A\right\} \\
= & \vee\left\{f^{2}(\mu)\left(f^{2}(i)\right) \wedge f^{2}(\mu)\right. \\
& \left.\left(f^{2}(y) \wedge f\left(f^{2}(i)\right)\right): x=f^{2}(y), \quad i \in A\right\} \\
= & \vee\left\{\mu\left(f^{2}(i)\right) \wedge \mu\left(f^{2}(y) \wedge f\left(f^{2}(i)\right)\right):\right. \\
& \left.x=f^{2}(y), \quad i \in A\right\} \\
= & \vee\left\{\mu\left(f^{2}(i)\right) \wedge \mu\left(x \wedge f\left(f^{2}(i)\right)\right): \quad f^{2}(i) \in A\right\} \\
\leq & \widetilde{\mu}(x) .
\end{aligned}
$$

Hence, $f^{2}(\widetilde{\mu}) \subseteq \widetilde{\mu}$, i.e., $\widetilde{\mu} \in \mathscr{F} \mathscr{I}_{2}(A)$.

Since $\mu \subseteq \widetilde{\mu}=\xi(\mu), \quad \xi \quad$ is extensive. Also, let $\mu, \nu \in \mathscr{F}_{2}(A)$ such that $\mu \subseteq \nu$. Then, 


$$
\begin{aligned}
\xi(\mu)(x) & =\widetilde{\mu}(x) \\
& =\bigvee\{\mu(i) \wedge \mu(x \wedge f(i)), \quad i \in A\} \\
& \leq \bigvee\{\nu(i) \wedge \nu(x \wedge f(i)), \quad i \in A\} \\
& =\widetilde{v}(x) \\
& =\xi(\mu)(x) .
\end{aligned}
$$

Thus, $\xi(\mu) \subseteq \xi(\nu)$, and hence, $\xi$ is isotone. Finally, we show that $\xi$ is idempotent.

Let $x \in A$. Then,

$$
\begin{aligned}
\xi(\xi(\mu))(x)= & \vee\{\xi(\mu)(s) \wedge \xi(\mu)(x \wedge f(s)): \quad s \in A\} \\
= & \vee\{\bigvee\{\mu(i) \wedge \mu(s \wedge f(i)): \quad i \in A\} \\
& \wedge \vee\{\mu(j) \wedge \mu(x \wedge f(s) \wedge f(j)): \quad j \in A\}: s \in A\} \\
= & \vee\{\mu(i) \wedge \mu(s \wedge f(i)) \wedge \mu(j) \\
& \wedge \mu(x \wedge f(s) \wedge f(j)): \quad s, i, j \in A\} \\
\leq & \vee\left\{\mu\left(f^{2}(i)\right) \wedge \mu((s \wedge f(i)) \vee j)\right. \\
& \wedge \mu(x \wedge f(s) \wedge f(j)): \quad s, i, j \in A\} \\
\leq & \vee\left\{\mu\left(x \wedge f(j) \wedge f^{2}(i)\right) \wedge \mu((s \wedge f(i)) \vee j)\right. \\
& \wedge \mu(x \wedge f(s) \wedge f(j)): \quad s, i, j \in A\} \\
\leq & \vee\left\{\mu\left(x \wedge f(j) \wedge f^{2}(i)\right) \vee(x \wedge f(s) \wedge f(j))\right. \\
& \wedge \mu((s \wedge f(i)) \vee j): \quad s, i, j \in A\} \\
= & \vee\{\mu(x \wedge f((s \wedge f(i)) \vee j)) \\
& \wedge \mu((s \wedge f(i)) \vee j): \quad s, i, j \in A\} \\
\leq & \xi(\mu)(x) .
\end{aligned}
$$

Thus, $\xi(\xi(\mu)) \subseteq \xi(\mu)$. As $\xi(\mu) \subseteq \xi(\xi(\mu))$, and we have $\xi(\xi(\mu))=\xi(\mu)$. Therefore, $\xi$ is idempotent. This proves the result.

\section{Smallest and Largest $L$-Fuzzy Congruences with a Given $L$-Fuzzy Kernel Ideal}

In this section, we describe the smallest and the largest $L$-fuzzy congruences on an Ockham algebra having a given $L$-fuzzy kernel ideal.

From Lemmas 8 and 9, we can easily observed that, for any kernel $L$-fuzzy ideal $\mu$ the smallest $L$-fuzzy congruence on $A$ with kernel $\mu$ is $\Omega_{L}(\mu)$ which is given by

$$
\begin{aligned}
& \Omega_{L}(\mu)(x, y) \\
& \quad=\vee\left\{\mu(a) \wedge(f(\mu))^{\uparrow}(b):(x \vee a) \wedge b=(y \vee a) \wedge b\right\} .
\end{aligned}
$$

We can in fact establish the following simpler version.
Theorem 6. If $\mu \in \mathscr{F}_{\mathscr{I}_{K}}(A)$, then the smallest L-fuzzy congruence on A with kernel $\mu$ is the L-fuzzy relation $\Phi_{L}(\mu)$ is given by

$$
\begin{aligned}
\Phi_{L}(\mu)(x, y) & =\vee\{\mu(i):(x \vee i) \wedge f(i) \\
& =(y \vee i) \wedge f(i), i \in A\} \text { for all } x \in A .
\end{aligned}
$$

Proof. To prove it enough to show that $\Phi_{L}(\mu)=\Omega_{L}(\mu)$, since $\mu(i) \leq f^{-1}(f(\mu))(i)=f(\mu)(f(i))$, we have

$$
\begin{aligned}
\Phi_{L}(\mu)(x, y) & =\vee\{\mu(i):(x \vee i) \wedge f(i)=(y \vee i) \wedge f(i), \quad i \in A\} \\
& =\vee\{\mu(i) \wedge f(\mu)(f(i)):(x \vee i) \wedge f(i) \\
& =(y \vee i) \wedge f(i), \quad i \in A\} \\
& \leq \Omega_{L}(\mu)(x, y) .
\end{aligned}
$$

Hence, $\Phi_{L}(\mu) \subseteq \Omega_{L}(\mu)$.

Since $\mu$ is kernel $L$-fuzzy ideal, 


$$
\begin{aligned}
\Omega_{L}(\mu)(x, y)= & \vee\left\{\mu(i) \wedge(f(\mu))^{\uparrow}(j):(x \vee i)\right. \\
& \wedge j=(y \vee i) \wedge j, \quad i, j \in A\}, \\
\Omega_{L}(\mu)(x, y)= & \vee\left\{\mu(i) \wedge(f(\mu))^{\uparrow}(j):(x \vee i)\right. \\
& \wedge j=(y \vee i) \wedge j, \quad i, j \in A\} \\
= & \vee\{\mu(i) \wedge \vee\{\mu(k): f(k) \leq(j)\}:(x \vee i) \\
& \wedge j=(y \vee i) \wedge j, \quad i, j \in A\} \\
= & \vee\{\mu(i) \wedge \mu(k):(x \vee i) \wedge f(k) \\
= & (y \vee i) \wedge f(k), \quad i, k \in A\} \\
\leq & \vee\{\mu(i \vee k):(x \vee i \vee k) \wedge f(i \vee k) \\
= & (y \vee i \vee k) \wedge f(i \vee k), \quad i, k \in A\} \\
= & \Phi_{L}(\mu)(x, y) .
\end{aligned}
$$

Hence, $\Omega_{L}(\mu)=\Phi_{L}(\mu)$. Thus, $\Phi_{L}(\mu)$ is the smallest $L$-fuzzy congruence with kernel $\mu$.

Having described the smallest $L$-fuzzy congruence on $A$ with a given kernel $L$-fuzzy ideal, we now proceed to determine the largest such $L$-fuzzy congruence.

For a given $L$-fuzzy ideal $\mu$ of $A$, each $a \in A$, and for each $n \in \mathbb{N}$, consider the $L$-fuzzy subset of $A$ defined by

$$
W_{a, n}^{\mu}(x)=\mu\left(f^{n}(a) \wedge x\right) \text { for all } x \in A,
$$

and an $L$-fuzzy binary relation $\Theta_{n}^{\mu}$ on $A$ defined by, for all

$$
a, b \in A \Theta_{n}^{\mu}(a, b)=\sup \left\{W_{a, n}^{\mu}(x) \wedge W_{b, n}^{\mu}(x): \quad x \in A\right\} .
$$

Lemma 13. $\Theta_{n}^{\mu}$ is a lattice L-fuzzy congruence of $A$ and $\cap_{n \in \mathbb{N}} \Theta_{n}^{\mu}$ is an L-fuzzy congruence of $A$.

Proof. For all $a, x \in A$,

$$
\begin{aligned}
\Theta_{n}^{\mu}(a, a) & =\sup \left\{W_{a, n}^{\mu}(x) \wedge W_{b, n}^{\mu}(x): \quad x \in A\right\} \\
& =\sup \left\{\mu\left(f^{n}(a) \wedge x\right) \wedge \mu\left(f^{n}(b) \wedge x\right): \quad x \in A\right\} \\
& \geq \mu\left(f^{n}(a) \wedge 0\right) \wedge \mu\left(f^{n}(b) \wedge 0\right) \\
& =\mu(0)=1 .
\end{aligned}
$$

This implies $\Theta_{n}^{\mu}$ is reflexive. Clearly, $\Theta_{n}^{\mu}$ is symmetric:

$$
\begin{aligned}
\Theta_{n}^{\mu}(a, b) \wedge \Theta_{n}^{\mu}(b, c)= & \sup \left\{W_{a, n}^{\mu}(x) \wedge W_{b, n}^{\mu}(x): \quad x \in A\right\} \\
& \wedge \sup \left\{W_{b, n}^{\mu}(y) \wedge W_{c, n}^{\mu}(y): \quad y \in A\right\} \\
= & \sup \left\{W_{a, n}^{\mu}(x) \wedge W_{b, n}^{\mu}(x) \wedge W_{b, n}^{\mu}(y)\right. \\
& \left.\wedge W_{c, n}^{\mu}(y): \quad x, y \in A\right\} \\
\leq & \sup \left\{W_{a, n}^{\mu}(x) \wedge W_{c, n}^{\mu}(y): \quad x, y \in A\right\} \\
\leq & \sup \left\{W_{a, n}^{\mu}(x \wedge y) \wedge W_{c, n}^{\mu}(x \wedge y): \quad x, y \in A\right\} \\
= & \Theta_{n}^{\mu}(a, c) .
\end{aligned}
$$

This implies $\Theta_{n}^{\mu}$ is transitive.

$$
\begin{aligned}
\Theta_{n}^{\mu}(a \vee c, b \vee d)= & \sup \left\{W_{a \vee c, n}^{\mu}(x) \wedge W_{b \vee d, n}^{\mu}(x): \quad x \in A\right\} \\
= & \sup \left\{\mu\left(f^{n}(a \vee c) \wedge x\right) \wedge \mu\left(f^{n}(b \vee d) \wedge x\right): \quad x \in A\right\} \\
& \cdot \operatorname{If} n-\text { even } \\
= & \sup \left\{\mu\left(\left(f^{n}(a) \vee f^{n}(c)\right) \wedge x\right)\right. \\
& \left.\wedge \mu\left(\left(f^{n}(b) \vee f^{n}(d)\right) \wedge x\right): \quad x \in A\right\} \\
= & \sup \left\{\mu\left(\left(f^{n}(a) \wedge x\right) \vee\left(f^{n}(c) \wedge x\right)\right)\right. \\
& \left.\wedge \mu\left(\left(f^{n}(b) \wedge x\right) \vee\left(f^{n}(d) \wedge x\right)\right): \quad x \in A\right\} \\
\geq & \sup \left\{\mu\left(f^{n}(a) \wedge x\right) \wedge \mu\left(f^{n}(c) \wedge x\right)\right. \\
& \left.\wedge \mu\left(f^{n}(b) \wedge x\right) \wedge \mu\left(f^{n}(d) \wedge x\right): \quad x \in A\right\} \\
= & \sup \left\{\mu\left(f^{n}(a) \wedge x\right) \wedge \mu\left(f^{n}(b) \wedge x\right)\right\} \\
& \wedge \sup \left\{\mu\left(f^{n}(c) \wedge x\right) \wedge \mu\left(f^{n}(d) \wedge x\right): \quad x \in A\right\} \\
= & \sup \left\{W_{a, n}^{\mu}(x) \wedge W_{b, n}^{\mu}(x):: \quad x \in A\right\} \\
& \wedge \sup \left\{W_{c, n}^{\mu}(x) \wedge W_{d, n}^{\mu}(x): \quad x \in A\right\} \\
= & \Theta_{n}^{\mu}(a, b) \wedge \Theta_{n}^{\mu}(c, d) .
\end{aligned}
$$

Also, if $n$ is odd

$$
\begin{aligned}
\Theta_{n}^{\mu}(a \vee c, b \vee d)= & \sup \left\{\mu\left(f^{n}(a) \wedge f^{n}(c) \wedge x\right)\right. \\
& \left.\wedge \mu\left(f^{n}(b) \wedge f^{n}(d) \wedge x\right): \quad x \in A\right\} \\
\geq & \sup \left\{\mu\left(\left(f^{n}(a) \wedge x\right)\right) \wedge \mu\left(\left(f^{n}(b) \wedge x\right)\right): \quad x \in A\right\} \\
= & \Theta_{n}^{\mu}(a, b) .
\end{aligned}
$$

Similarly, $\quad \Theta_{n}^{\mu}(a \vee c, b \vee d) \geq \Theta_{n}^{\mu}(c, d)$. This implies $\Theta_{n}^{\mu}(a \vee c, b \vee d) \geq \Theta_{n}^{\mu}(a, b) \wedge \Theta_{n}^{\mu}(c, d)$. Hence, $\Theta_{n}^{\mu}$ is compatible with $\vee$. Similarly, $\Theta_{n}^{\mu}$ is compatible with $\wedge$. Hence, $\Theta_{n}^{\mu}$ is a lattice $L$-fuzzy congruence of $A$.

Put $\Theta^{\mu}=\cap_{n \in \mathbb{N}} \Theta_{n}^{\mu}$. Then, clearly $\Theta^{\mu}$ is a lattice $L$-fuzzy congruence of $A$. Next, we show that, for any $a, b \in A$,

$$
\begin{aligned}
\Theta^{\mu}(f(a), f(b)) \geq & \Theta^{\mu}(a, b), \\
\Theta^{\mu}(f(a), f(b))= & \left(\bigcap_{n \in \mathbb{N}} \Theta_{n}^{\mu}\right)(f(a), f(b)) \\
= & \inf _{n \in \mathbb{N}}\left(\Theta_{n}^{\mu}(f(a), f(b))\right. \\
= & \inf _{n \in \mathbb{N}}\left\{\Theta_{n}^{\mu}(f(a), f(b))\right\} \\
= & \inf _{n \in \mathbb{N}}\left\{\operatorname { s u p } \left\{\mu\left(f\left(f^{n}(a)\right) \wedge x\right)\right.\right. \\
& \left.\left.\wedge \mu\left(f\left(f^{n}(b)\right)\right): \quad x \in A\right\}\right\} \\
\geq & \inf _{n \in \mathbb{N}}\left\{\operatorname { s u p } \left\{\mu\left(f^{n}(a) \wedge x\right)\right.\right. \\
& \left.\left.\wedge \mu\left(f^{n}(b) \wedge x\right): \quad x \in A\right\}\right\} \\
= & \inf _{n \in \mathbb{N}}\left(\theta_{n}^{\mu}(a, b)\right) \\
= & \theta^{\mu}(a, b) .
\end{aligned}
$$

Thus, $\theta^{\mu}(a, b) \leq \theta^{\mu}(f(a), f(b))$. Hence, $\theta^{\mu}$ is an $L$-fuzzy congruence of $A$. 
In the next theorem, we describe the largest $L$-fuzzy congruence on $A$ containing a given $L$-fuzzy ideal $\mu$ as its kernel.

Theorem 7. If $\mu \in \mathscr{F}_{K} \mathscr{I}_{K}(A)$, then the largest L-fuzzy congruence on A with kernel $\mu$ is $\Theta^{\mu}$.

Proof. First, we prove that $\operatorname{ker}^{\mu}=\mu$. For any $x \in A$,

$$
\begin{aligned}
\mu(x) & \leq \mu\left(f^{n}(x)\right) \\
& \leq \mu\left(f^{n}(x) \wedge a\right) \\
& =\mu\left(f^{n}(x) \wedge a\right) \\
& =W_{x, n}^{\mu}(a) .
\end{aligned}
$$

Since $W_{0, n}^{\mu}(a)=1$. This implies

$$
\begin{aligned}
\mu(x) & \leq W_{x, n}^{\mu}(a) \wedge W_{0, n}^{\mu}(a) \\
& \leq \inf _{n \in N}\left\{\sup \left\{W_{x, n}^{\mu}(a) \wedge W_{0, n}^{\mu}(a): \quad a \in A\right\}\right\} \\
& =\inf _{n \in N}\left(\theta_{n}^{\mu}(x, 0)\right) \\
& =\left(\theta^{\mu}(x, 0)\right)=\operatorname{ker} \theta^{\mu}(x) .
\end{aligned}
$$

This implies $\mu \subseteq \operatorname{ker} \theta^{\mu}$.

Conversely,

$$
\begin{aligned}
\operatorname{ker} \theta^{\mu}(x) & =\inf _{n \in N}\left\{\sup \left\{W_{x, n}^{\mu}(a) \wedge W_{0, n}^{\mu}(a): \quad a \in A\right\}\right\} \\
& \leq \sup \left\{W_{x, n}^{\mu}(a) \wedge W_{0, n}^{\mu}(a): \quad a \in A\right\} \\
& =\sup \left\{\left(\mu\left(f^{n}(x) \wedge a\right) \wedge \mu\left(f^{n}(0) \wedge a\right): \quad a \in A\right\}\right. \\
& \leq \mu(x) .
\end{aligned}
$$

This implies $\operatorname{ker} \theta^{\mu} \subseteq \mu$. Hence, $\operatorname{ker} \theta^{\mu}=\mu$.

Finally, we prove that $\theta^{\mu}$ is the largest $L$-fuzzy congruence of $A$. Now, let $\Phi$ be any $L$-fuzzy congruence on $A$ with kernel $\mu$. We show that $\Phi \subseteq \Theta^{\mu}$. For $a, b, x \in A$, $\Phi(a, 0)=\operatorname{ker} \Phi(a)=\mu(a) \leq \mu(a \wedge x)=W_{a, 0}^{\mu}(x)$ and $\Phi(0, b)$ $=\operatorname{ker} \Phi(b)=\mu(b) \leq \mu(b \wedge x)=W_{b, 0}^{\mu}(x)$. This implies $\Phi(a, 0)$ $\wedge \Phi(0, b) \leq W_{a, 0}^{\mu}(x) \wedge W_{b, 0}^{\mu}(x) \leq \sup \left\{W_{a, n}^{\mu}(x) \wedge W_{b, n}^{\mu}(x): x \epsilon\right.$ A\} for all $n \in W$.

It follows that

$$
\begin{aligned}
\Phi(a, b) & \leq \inf _{n \in W}\left\{\sup \left\{W_{a, n}^{\mu}(x) \wedge W_{b, n}^{\mu}(x): \quad x \in A\right\}\right\} \\
& =\theta^{\mu}(a, b) .
\end{aligned}
$$

Hence, $\theta^{\mu}$ is the largest $L$-fuzzy congruence on $A$ having $\mu$ as a kernel.

\section{Conclusion}

In this paper, we study $L$-fuzzy congruences and $L$-fuzzy kernel ideals in Ockham algebras and investigate their properties. A set of equivalent conditions is derived for an $L$-fuzzy ideal $\mu$ of $A$ to become an $L$-fuzzy kernel ideal. We obtain the smallest, respectively, the largest $L$-fuzzy congruences on $A$ having a given $L$-fuzzy ideal as a kernel and describe it using algebraic operations in a fuzzy setting.
It is under investigation by the authors to characterize those subvarieties of the class of Ockham algebras using $L$-fuzzy congruence relations.

\section{Data Availability}

No data were used to support the findings of this study.

\section{Conflicts of Interest}

The authors declare that there are no conflicts of interest regarding to the publication of this paper.

\section{References}

[1] L. A. Zadeh, "Fuzzy sets," Information and Control, vol. 8, no. 3, pp. 338-353, 1965.

[2] P. Kaur and T. Chaira, "A novel fuzzy approach for segmenting medical images," Soft Computing, vol. 25, no. 5, pp. 3565-3575, 2021.

[3] J. Priyadharsini and P. Balasubramaniam, "Existence of fuzzy fractional stochastic differential system with impulses," Computational and Applied Mathematics, vol. 39, pp. 1-21, 2020.

[4] R. Verma and B. D. Sharma, "Fuzzy generalized prioritized weighted average operator and its application to multiple attribute decision making," International Journal of Intelligent Systems, vol. 29, no. 1, pp. 26-49, 2014.

[5] R. Verma and B. D. Sharma, "A measure of inaccuracy between two fuzzy sets," Cybernetics and Information Technologies, vol. 11, no. 2, pp. 13-23, 2011.

[6] R. Verma and S. Maheshwari, "A new measure of divergence with its application to multi-criteria decision making under fuzzy environment," Natural Computing and Applications, vol. 28, no. 8, pp. 2323-2350, 2017.

[7] A. Rosenfeld, "Fuzzy groups," Journal of Mathematical Analysis and Applications, vol. 35, no. 3, pp. 512-517, 1971.

[8] B. A. Alaba and T. G. Alemayehu, "Closure fuzzy ideals of MSalgebras," Annals of Fuzzy Mathematics and Informatics, vol. 16, no. 2, pp. 247-260, 2018.

[9] B. A. Alaba and T. G. Alemayehu, "Fuzzy ideals in demipseudocomplemented MS-algebras," Annals of Fuzzy Mathematics and Informatics, vol. 18, no. 2, pp. 123-143, 2019.

[10] B. A. Alaba and T. G. Alemayehu, " $\beta$ - fuzzy filters of MSalgebras," Korean Journal of Mathematics, vol. 27, pp. 595612, 2019.

[11] B. A. Alaba and W. Z. Norahun, "Fuzzy annihilator ideals in distributive lattices," Annals of Fuzzy Mathematics and Informatics, vol. 16, no. 2, pp. 191-200, 2018.

[12] B. A. Alaba and W. Z. Norahun, " $\alpha$-fuzzy ideals and space of prime $\alpha$-fuzzy ideals in distributive lattices," Annals of Fuzzy Mathematics and Informatics, vol. 17, no. 2, pp. 147-163, 2019.

[13] W. Z. Norahun, " $\mu$-Fuzzy filters in distributive lattices," Advances in Fuzzy Systems, vol. 2020, Article ID 8841670, 9 pages, 2020.

[14] B. A. Alaba, M. A. Taye, and D. A. Engidaw, "L-fuzzy ideals of a poset," Annals of Fuzzy Mathematics and Informatics, vol. 16, no. 3, pp. 285-299, 2018.

[15] B. A. Alaba, M. A. Taye, and D. A. Engidaw, " $L$-fuzzy prime ideals and maximal $L$-fuzzy ideals of a poset," Annals of Fuzzy Mathematics and Informatics, vol. 18, no. 1, pp. 1-13, 2019.

[16] B. A. Alaba and G. M. Addis, " $L$-fuzzy ideals in universal algebras," Annals of Fuzzy Mathematics and Informatics, vol. 17, no. 1, pp. 31-39, 2019. 
[17] B. A. Alaba and G. M. Addis, " $L$-Fuzzy prime ideals in universal algebras," Advances in Fuzzy Systems, vol. 2019, Article ID 5925036, 7 pages, 2019.

[18] B. A. Alaba and G. M. Addis, " $L$-Fuzzy semi-prime ideals in universal algebras," Korean Journal of Mathematics, vol. 27, no. 2, pp. 327-340, 2019.

[19] K. T. Atanassov, "Intuitionistic fuzzy sets," Fuzzy Sets and Systems, vol. 20, no. 1, pp. 87-96, 1986.

[20] M. Bhowmik, T. Senapati, and M. Pal, "Intuitionistic $L$-fuzzy ideals of BG -algebras," Afrika Matematika, vol. 25, no. 3, pp. 577-590, 2014.

[21] T. Senapati, Y. B. Jun, and K. P. Shum, "Cubic intuitionistic subalgebras and closed cubic intuitionistic ideals of B-algebras," Journal of Intelligent \& Fuzzy Systems, vol. 36, no. 2, pp. 1563-1571, 2019.

[22] T. Senapati, Y. B. Jun, and K. P. Shum, "Cubic intuitionistic implicative ideals of BCK-algebras," Proceedings of the $\mathrm{Na}$ tional Academy of Sciences, India Section A: Physical Sciences, vol. 91, no. 2, pp. 273-282, 2021.

[23] M. Samhan, "Fuzzy congruences on semigroups," Information Sciences, vol. 74, no. 1-2, pp. 165-175, 1993.

[24] Y. Tan, "Fuzzy congruences on a regular semigroup," Fuzzy Sets and Systems, vol. 117, no. 3, pp. 447-453, 2001.

[25] T. K. Dutta and B. K. Biswas, "Fuzzy congruence and quotient semiring of a semiring," Journal of Fuzzy Mathematics, vol. 4, pp. 737-748, 1996.

[26] J. P. Kim and D. R. Bae, "Fuzzy congruences in groups," Fuzzy Sets and Systems, vol. 85, no. 1, pp. 115-120, 1997.

[27] M. Kondo, "Fuzzy congruences on groups," Quasigroups and Related Systems, vol. 11, pp. 59-70, 2004.

[28] N. Kuroki, "Fuzzy congruences and fuzzy normal subgroups," Information Sciences, vol. 60, no. 3, pp. 247-259, 1992.

[29] M. A. Samhan and T. M. G. Ahsanullah, "Fuzzy congruences on groups and rings," International Journal of Mathematics and Mathematical Sciences, vol. 17, no. 3, pp. 469-473, 1994.

[30] X. Zhou, D. Xiang, and J. Zhan, "Quotient rings via fuzzy congruence relations," Italian Journal of Pure and Applied Mathematics, vol. 33, pp. 411-424, 2014.

[31] T. K. Dutta and B. K. Biswas, "On fuzzy congruence of a nearring module," Fuzzy Sets and Systems, vol. 112, no. 2, pp. $343-348,2000$.

[32] S. K. Shoara, R. A. Borzooeib, and R. Moradian, "Fuzzy congruence relation generated by a fuzzy relation in vector spaces," Journal of Intelligent and Fuzzy Systems, vol. 32, 2018.

[33] U. M. Swamy and D. V. Raju, "Fuzzy ideals and congruences of lattices," Fuzzy Sets and Systems, vol. 95, no. 2, pp. 249-253, 1998.

[34] Bo. Yuan and W. Wu, "Fuzzy ideals on a distributive lattice," Fuzzy Sets and Systems, vol. 35, pp. 231-240, 1990.

[35] B. A. Alaba and G. M. Addis, "Fuzzy congruence relations on almost distributive lattices," Annals of Fuzzy Mathematics and Informatics, vol. 14, no. 3, pp. 315-330, 2017.

[36] B. A. Alaba, M. A. Taye, and T. G. Alemayehu, "Fuzzy congruences on MS-algebras," Journal of Mathematics and Informatics, vol. 15, pp. 49-57, 2019.

[37] G. M. Addis, N. Kausar, M. Munir, and Y. M. Chung, "The commutator of fuzzy congruence in universal algebras," International Journal of Computational Intelligence Systems, vol. 14, pp. 1322-1336, 2021.

[38] V. Murali, "Fuzzy congruence relations," Fuzzy Sets and Systems, vol. 41, no. 3, pp. 359-369, 1991.

[39] M. A. Samhan, "Fuzzy quotient algebras and fuzzy factor congruences," Fuzzy Sets and Systems, vol. 73, no. 2, pp. 269-277, 1995.
[40] J. Berman, "Distributive lattices with an additional unary operation," Aequationes Math, vol. 16, pp. 165-171, 1974.

[41] T. S. Blyth and H. J. Silva, "Congruence kernels in Ockham algebras," Algebra Universalis, vol. 78, no. 1, pp. 55-65, 2017.

[42] T. S. Blyth and J. C. Varlet, Ockham Algebras, Oxford University Press, Oxford, UK, 1994.

[43] J. N. Mordeson and D. S. Malik, Fuzzy Commutative Algebra, World Science Publication, Singapore, 1998.

[44] J. A. Goguen, "L-fuzzy sets," Journal of Mathematical Analysis and Applications, vol. 18, no. 1, pp. 145-174, 1967.

[45] V. Murali, "Fuzzy equivalence relations," Fuzzy Sets and Systems, vol. 30, no. 2, pp. 155-163, 1989. 